Energy Research Journal 1 (2): 91-95, 2010

ISSN 1949-0151

(C) 2010 Science Publications

\title{
Effects of Aspect Ratios and Number of Meandering Turns on Performance Limit of an Inclined Closed-Loop Oscillating Heat Pipe
}

\author{
N. Panyoyai, P. Terdtoon and P. Sakulchangsatjatai \\ Department of Mechanical Engineering, Faculty of Engineering, \\ Chiang Mai University, Chiang Mai, Thailand 50200
}

\begin{abstract}
Problem statement: The purpose of this research was to determine the effects of aspect ratios and number of meandering turns on performance limit of an inclined closed-loop oscillating heat pipe. Approach: The Closed-Loop Oscillating Heat Pipe (CLOHP) was made of a long copper capillary tube. The geometrical sizes, which were the variable parameters of this study, were as follows; the internal diameter of $0.66,1.06$ and $2.03 \mathrm{~mm}$, the evaporator section length of 50, 100 and $150 \mathrm{~mm}$, the adiabatic and condenser section length of each set was equaled to the evaporator length and the numbers of meandering turn of 5,10 and 15 turns. The experiments were conducted by setting the inclination angles at $0-90^{\circ}$ adjusted by $10^{\circ}$, there totally were 9 sets and each set was tested with R123 as working fluid. The adiabatic temperature was controlled at $50 \pm 5^{\circ} \mathrm{C}$. The low-voltage and high-current power transformer was used as the heat source and heat transfer rate was obtained by using calorific method at the condenser section. Results: It was found from the experiments that, in case of the CLOHP with 10 turns and the internal diameter of $2.03 \mathrm{~mm}$, the maximum heat flux increases from 10-35.5 $\mathrm{kW} \mathrm{m}^{-2}$ when the aspect ratio decreases from 151.5-49.26 and the highest maximum heat flux occurs at inclination angle about $70-90^{\circ}$. Conclusion: The result indicated that the aspect ratio, the ratio of evaporator length by internal diameter and number of meandering turns significantly affect the maximum heat flux and inclination angle.
\end{abstract}

Key words: Inclination, closed-loop oscillating heat pipe, performance limit

\section{INTRODUCTION}

Recently new inventive technologies consist of smaller electronic devices for sustainable well-being. On contrary, they are more efficient. Specifically, they transfer more heat while they have less cooling area and cause more accumulative heat on such devices. Thus, it is important to develop the small heat exchanger to dissipate that heat. The CLOHP, therefore, becomes a best choice at present. Since the CLOHP is a small heat transfer device with very high thermal conductivity.

The CLOHPs are made from a long copper capillary tube, bent into undulating tube and connected each other ends into closed-loop form and no internal wick structure is required. The structures are shown in Fig. 1.

The heat transfer mechanism of CLOHP begins while one end of the bundle of the heat pipe is subjected into heat or high temperature. The working fluid, which is in liquid plug and vapor bubble form, will evaporate, expand and move toward to cooler section. Then, vapor bubble will condense collapse and release the heat to heat sink. Since the evaporation and collapsing of vapor bubble as mentioned above is the main mechanism by which the working fluid can circulate and continuously transfer the heat in cycle. (Soponpongpipat et al., 2006) It is clearly known that the working fluid is able to form itself into liquid plug alternates with vapor bubble for entire tube if the diameter of the capillary tube used to make the oscillating heat pipe does not exceed the critical diameter in Eq. 1. (Maezawa, 2000):

$$
\mathrm{D}_{\mathrm{i}, \mathrm{crit}}=2 \sqrt{\frac{\sigma}{\rho_{\mathrm{L}} \mathrm{g}}}
$$
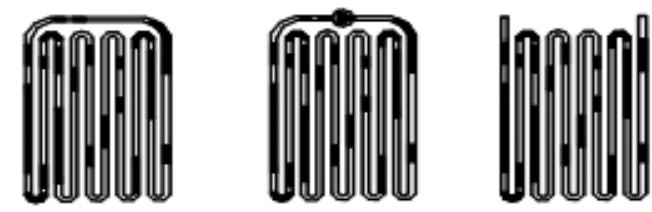

Fig. 1: Three types of oscillating heat pipes

Corresponding Author: N. Panyoyai, Department of Mechanical Engineering, Faculty of Engineering, Chiang Mai University, Chiang Mai, Thailand 50200 Tel: +66 53 944144/ext.911 Fax: +66 53226014 
When the CLOHP is supplied with heat, higher evaporation rate of the working fluid and its vapor velocity and pressure can be realized. While it reaches a specified condition, the working fluid in liquid phase is inadequate for evaporation caused by input heat rate. Subsequently, there is a dryout occurring inside tube surface at evaporator section. In some cases, thus, heat transferred by the CLOHP will decrease and the tube could be damaged if more heat is continuously supplied. Therefore, the heat flux which the CLOHP work properly just before its dryout is defined as "performance limit". There are a number of studies on heat transfer characteristics of the CLOHP only at normal state. Otherwise, there is few of study at critical state e.g., effect of working fluids on heat transfer characteristics of CLOHP at critical state. KammuangLue et al. (2004) found that, when the latent heat of working fluid increases, the average maximum heat flux increased. Sakulchangsatjatai et al. (2007) found that, when the evaporator section length increases the maximum heat flux decreases but when the internal diameter and meandering turn increases the maximum heat flux increases. Moreover inclination angle significantly effects to normal state heat transfer of CLOHP at normal operation. In order to the clarify knowledge from the past study and to prevent direct damage on CLOHP, it is important to study the effect of many parameters on performance limits of an inclined CLOHP. The results from this study will be very useful with the referable basic data in actual application designs. Therefore, the objectives of this study are to experimentally investigate the effect of aspect ratio and the number of meandering turns on performance limits of an inclined CLOHP.

\section{MATERIALS AND METHODS}

Figure 2 shows schematic diagram of the experimental setup in this study. The CLOHP was made from a long copper capillary tube with the inner diameter of $0.66,1.06$ and $2.03 \mathrm{~mm}$ and bent into 5, 10 and 15 turns. Then, both ends were connected to form the loop. Two copper bus bars were welded, one at the top and the other at the bottom of evaporator section as electric bus bars for equal heat generation. The distance between these two bus bars plates equaled to evaporator section length, $\mathrm{L}_{\mathrm{e}}$ which is $100 \mathrm{~mm}$ for this study. There were totally 9 oscillating heat pipe sets as shown in Table 1 . The experiments were conducted by setting the inclination angles at $0-90^{\circ}$ with the increment of $10^{\circ}$. The adiabatic and condenser section length of each set was equaled to the evaporator length in order to eliminate the effect of heat flux transformation. R123 was selected as working fluid. The filling ratio was $50 \%$ by total volume.
The low-voltage and high-current power transformer was used as the heat source by supplying the electrical current to the upper bus bar. Then, the current passed through the capillary tube bundle to the lower bus bar. Due to the electrical resistance of the copper tube, thus, the evaporator section became heater by itself. The quantity of heat was controlled by voltage adjustment. The whole condenser section was in the cooling jacket. The cooling medium was the solution of water and ethylene-glycol with 1:1 mixing volume ratio. This cooling medium was circulated to transfer heat from condenser section to the heat sink which was the cooling bath. The flow rate was measured by a rotameter (Platon, PGB411, accuracy $\pm 0.1 \mathrm{~L} \mathrm{~min}^{-1}$ ). The temperature at specified points was monitored by a data logger (Brainchild, VR18, accuracy $\pm 0.1{ }^{\circ} \mathrm{C}$ ). 17 Chromel-Alumel thermocouples (Omega, Type K, accuracy $\pm 0.5^{\circ} \mathrm{C}$ ) were installed on the outer surface of the capillary tube for measuring the variation of temperature at every parts of the heat pipe. They consisted of 8 points on the middle of each tube in evaporator section, 3 points in adiabatic section and 3 points in condenser section. Additional 2 thermocouples on each inlet and outlet tube of cooling jacket for measuring the variation of cooling medium temperature in order to calculate the heat flux at specific time by mean of the calorific method as Eq. 2:

$$
\dot{\mathrm{q}}=\frac{\dot{\mathrm{m}}_{\mathrm{c}} \mathrm{c}_{\mathrm{pc}}\left(\mathrm{T}_{\text {out }}-\mathrm{T}_{\mathrm{in}}\right)_{\mathrm{c}}}{\mathrm{A}_{\mathrm{c}}}
$$

Where:

$\dot{\mathrm{m}}_{\mathrm{c}}=$ The mass flow rate of cooling medium

$\mathrm{c}_{\mathrm{pc}} \quad=$ The specific heat of cooling Medium (water and ethylene-glycol)

$\left(\mathrm{T}_{\text {out }}-\mathrm{T}_{\text {in }}\right)_{\mathrm{c}}=$ The temperature different of cooling medium

$\mathrm{A}_{\mathrm{c}} \quad=$ All outer surface area of tube in condenser section

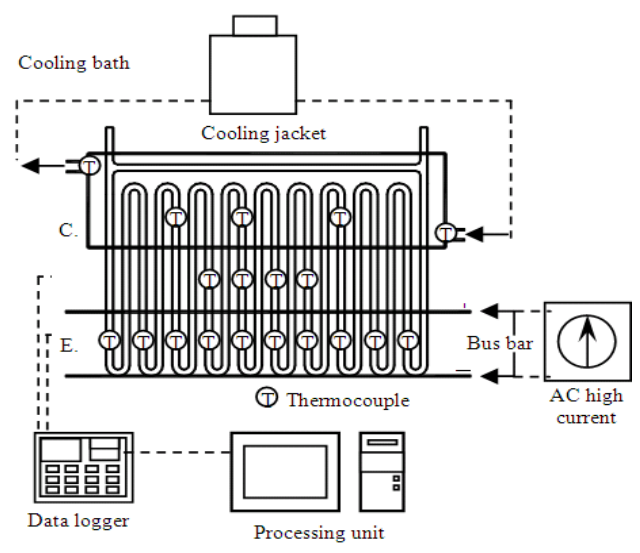

Fig. 2: The experimental setup 
Table1: Testing set $\left(\mathrm{R} 123, \mathrm{~L}_{\mathrm{e}}=100 \mathrm{~mm}\right)$

\begin{tabular}{llll}
\hline $\mathrm{D}_{\mathrm{i}}(\mathrm{mm})$ & & Turn $(\mathrm{N})$ & \\
\hline 0.66 & 5 & 10 & 15 \\
1.06 & 5 & 10 & 15 \\
2.03 & 5 & 10 & 15 \\
\hline
\end{tabular}

The experimental procedure was as follows. Heat was gradually supplied in a small step into evaporator section. In each step, the adiabatic temperature was controlled at $50 \pm 5^{\circ} \mathrm{C}$ (because this is the suitable working temperature and did not exceed the critical point of all chosen working fluids) by adjusting either the temperature or the flow rate of cooling medium. When system reached the steady state, the temperature of each point in evaporator section was recorded. If all temperatures changed consecutively, it showed that the heat pipe could transfer heat properly. Then, the heat was further increased. When the heat was higher than a certain level, one or more points at evaporator section rapidly rose in temperature show that the liquid film in inside wall of closed loop pulsating heat pipe subsequently becomes thinner and dryout occurs. It showed that the heat pipe has reached critical state. The maximum heat flux can be calculated by Eq. 2 and using data before critical state by measuring the temperature and the mass flux of the cooling medium on both inlet and outlet of the cooling jacket. Finally, the heat transfer characteristics at critical state will be achieved. The error of obtained heat flux could be calculated from Eq. 3 where $\mathrm{dq}$ was the error of the maximum heat flux, dm ${ }_{c}$ was the accuracy from measuring the mass flow rate of cooling medium, $\mathrm{dT}_{\mathrm{c}, \mathrm{in}}$ and $\mathrm{dT}_{\mathrm{c}, \text { out }}$ was the accuracy from measuring the inlet and outlet temperature respectively:

$$
\mathrm{dq}=\left(\begin{array}{l}
\left(\frac{\partial \mathrm{q}}{\partial \dot{\mathrm{m}}_{\mathrm{c}}} \mathrm{d}_{\mathrm{c}}\right)^{2}+\left(\frac{\partial \mathrm{q}}{\partial \mathrm{C}_{\mathrm{pc}}} \mathrm{dC}_{\mathrm{pc}}\right)^{2} \\
+\left(\frac{\partial \mathrm{q}}{\partial \mathrm{T}_{\mathrm{c}, \text { out }}} \mathrm{d}_{\mathrm{c}, \text { out }}\right)^{2}+\left(\frac{\partial \mathrm{q}}{\partial \mathrm{T}_{\mathrm{c}, \text { in }}} \mathrm{d}_{\mathrm{c}, \text { in }}\right)^{2}
\end{array}\right)^{2}
$$

\section{RESULTS}

Effect of aspect ratios: It can be found from the study that, when the aspect ratios increases, the maximum heat flux sharply decreases at inclination about $50-90^{\circ}$, as shown in Fig. 3. In case of the CLOHP with evaporator length of $100 \mathrm{~mm}$ and number of meandering turn of 15 turns, it can be seen that, when the aspect ratios increases from 49.3-151.5, the maximum heat flux decreases for all inclination angle (Fig. 4).

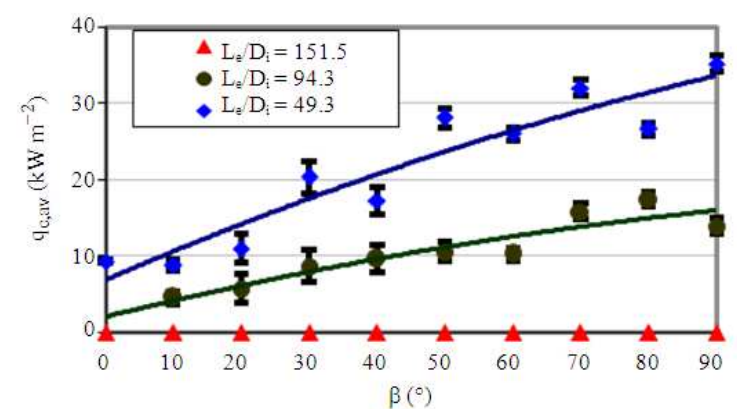

Fig. 3: Effect of inclination angle on maximum heat flux on each aspect ratio $\left(\mathrm{L}_{\mathrm{e}}=100 \mathrm{~mm}, \mathrm{~N}=15 \mathrm{R} 123\right)$

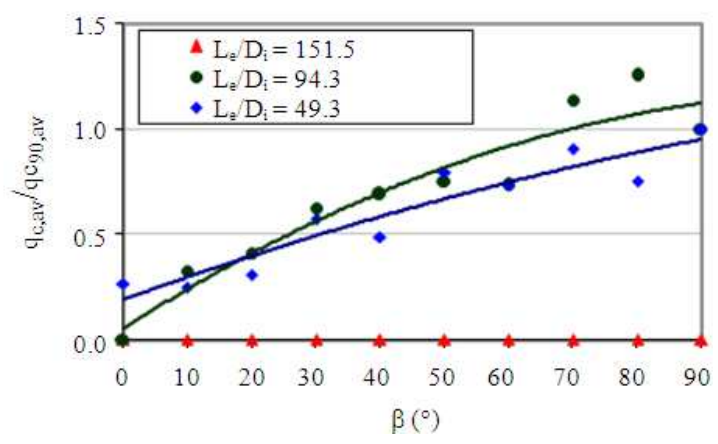

Fig. 4: Effect of inclination angle on $\mathrm{q}_{\mathrm{c}, \mathrm{av}} / \mathrm{qc}_{90, \mathrm{av}}$ on each aspect ratio $\left(\mathrm{L}_{\mathrm{e}}=100 \mathrm{~mm}, \mathrm{~N}=15 \mathrm{R} 123\right)$

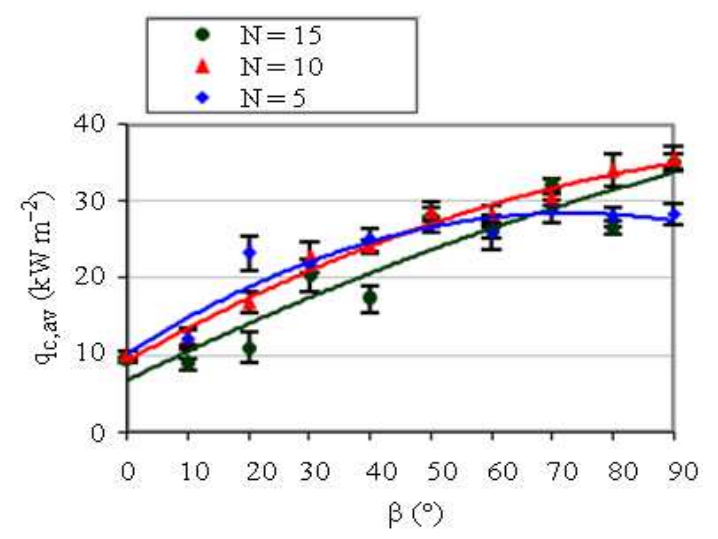

Fig. 5: Effect of inclination angle on maximum heat flux $\left(\mathrm{L}_{\mathrm{e}}=100 \mathrm{~mm}, \mathrm{D}_{\mathrm{i}}=2.03 \mathrm{R} 123\right)$

Experiments with 5 turns showed similar trend to those of 10 turns. When the aspect ratio increases, the maximum heat flux decreases. In case of 15 turns, the trends are fairly similar to 10 and 5 turns. It can be found that the highest ratio of heat flux average to maximum heat flux average at $90^{\circ}$ occurs at aspect ratio at 49.3 and inclination on vertical position to about $70^{\circ}$. Thurs, the trends all of studies are fairly closed. 


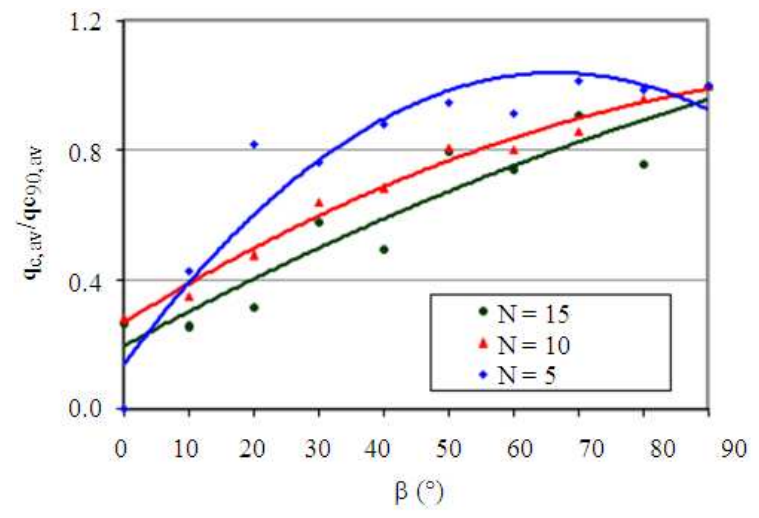

Fig. 6: Effect of inclination angle on $\mathrm{q}_{\mathrm{c}, \mathrm{av}} / \mathrm{qc}_{90, \mathrm{av}}$ on each meandering turns $\left(\mathrm{L}_{\mathrm{e}}=100 \mathrm{~mm}, \mathrm{D}_{\mathrm{i}}=2.03 \mathrm{R} 123\right)$

Effect of Inclination angle on maximum heat flux at each meandering turns: It can be found from the study that, when the Inclination angle increases, the maximum heat flux sharply increases, but the inclination angle does not effect to the meandering turns as shown in Fig. 5. For example, in case of the CLOHP with meandering turns of 5, 10 and 15 turns, evaporator length of $100 \mathrm{~mm}$, internal diameter of $2.03 \mathrm{~mm}$ and R123 as working fluid (Fig. 6), it can be seen that, when the inclination angle increases from 0 $90^{\circ}$, the maximum heat flux rather increases from 0 $28.4 \mathrm{~kW} \mathrm{~m}^{-2}$ for 5 turns, $10-35.5 \mathrm{~kW} \mathrm{~m}^{-2}$ for 10 turns and $9.3-35.2 \mathrm{~kW} \mathrm{~m}^{-2}$ for 15 turns respectively.

\section{DISCUSSION}

Effect of aspect ratios: The maximum heat flux vice versa varies with the aspect ratio because increasing the aspect ratio that mean internal diameter is decreased and likewise decrease in heat transfer and cross section areas inside the oscillating heat pipe. The maximum heat flux directly varies only with the internal diameter. It can be considered at the same input heat transfer that, the inside evaporation rate should be roughly the same. Since, the higher internal diameter heat pipe or, in turn, the more cross section area, thus, the vapor velocity is lower. The liquid film can transfer much more heat until the velocity reaches a certain value that causes the internal flow pattern changes into annular flow. The liquid film subsequently becomes thinner and dry out occurs. In a case of aspect ratio 151.5 can be seen that CLOHP cannot transfer heat all of inclination, because of dry out occurs.

Effect of inclination angle on maximum heat flux at each meandering turns: The highest maximum heat flux occurs. In the angle range of vertical position to about $70^{\circ}$ for all of sets. This might be because when inclination angle increases the gravity effect, which plays an important role in the movement of vapor plug, sharply increases.

In addition, these results are compared with the past study of the CLOHP at normal state conducted by Charoensawan et al. (2003). It can be found that the ratio of maximum heat flux at any angles to maximum heat flux at $90^{\circ}$ reaches its highest value at vertical position to about $70^{\circ}$ and the trends in both studies are fairly closed. Therefore the inclination angle has obvious effect on the maximum hear flux.

\section{CONCLUSION}

The effects of aspect ratios and number of meandering turns on maximum heat flux of an inclined CLOHP have been thoroughly investigated in this study. In the case of aspect ratio, it can be seen that, the highest maximum heat flux occurs at inclination angle about $70-90^{\circ}$ and aspect ratio is 49.3 .

In the case of number of meandering turns, it can be seen that, when number of meandering turns increases from 5-15 turns, number of meandering turns does not affect to the maximum heat flux.

In the case of inclination angle, it can be seen that, when the inclination angle increases from $0-90^{\circ}$, the maximum heat flux increases from $0-28.4 \mathrm{~kW} \mathrm{~m}^{-2}$ for 5 turns, $10-35.5 \mathrm{~kW} \mathrm{~m}^{-2}$ for 10 turns and $9.3-35.2 \mathrm{~kW} \mathrm{~m}^{-2}$ for 15 turns respectively. Moreover the highest maximum heat flux occurs at vertical position to about $70^{\circ}$.

\section{ACKNOWLEDGEMENT}

This research was conducted under Graduate school of Chiang Mai University and Maejo University Chiang Mai Thailand. The authors would like to express their sincere appreciation for all of the support provided.

\section{REFERENCES}

Charoensawan, P., S. Khandekar, M. Groll and P. Terdtoon, 2003. Closed loop pulsating heat pipes part: A Parametric Experimental Investigations. Applied Therm. Eng., 23: 2009-2020.

Kammuang-Lue, N., P. Charoensawan, S. Ritthidech, K. Budhajan and P. Terdtoon, 2004. Effect of working fluids on heat transfer characteristics of a closed-loop pulsating heat pipe at critical state. Proceedings of the 1st International Seminar on Heat Pipes and Heat Recovery Systems, Dec. 8-9, Kuala Lumpur, Malaysia, pp: 121-127. 
Katpradit, T., T. Wongratanaphisan, P. Terdtoon, P. Kamonpet, A. Polchai and A. Akbarzadeh, 2005. Correlation to predict heat transfer characteristics of a closed end oscillating heat pipe at critical state. Applied Therm. Eng., 25: 2138-2135.

Khandekar, S., P. Charoensawan, M. Groll and P. Terdtoon, 2003. Closed loop pulsating heat pipes part: $\mathrm{B}$ visualization and semi-empirical modeling. Applied Therm. Eng., 23: 2021-2033.

Maezawa, S., 2000. Heat Pipe: Its origin, development and present situation. Proceedings of the 6th International Symposium on Heat Pipe, Nov. 5-9, Chiang Mai, Thailand, pp: 1-13.

Panyoyai, N., P. Sakulchangsatjatai and P. Terdtoon, 2008. Effects of internal diameter and evaporator length on maximum heat flux of an inclined closedloop pulsating heat pipe. Proceedings of the 9th International Symposium on Heat Pipe, Nov. 17-20, Selangor, Malaysia, pp: 126-130.
Sakulchangsatjatai, P., N. Kammuang-Lue, P. Terdtoon and D.J. Mook, 2007. Effect of geometrical sizes on maximum heat flux of a vertical closed-loop pulsating heat pipe. Proceedings of the 14th International Conference on Heat Pipe, Apr. 22-27, Florianópolis, Brazil.

Soponpongpipat, N., P. Sakulchangsatjatai, M. Sornseub and P. Terdtoon, 2006. Time response model of operational mode of closed-loop oscillating heat pipe at normal operation. Proceedings of the 8th International Symposium on Heat Pipe, Sept. 2427, Kumamoto, Japan, pp: 291-296. 